\title{
Open Notes and Closed-Loop Communication: A Case of Factitious Disorder in the Age of Transparent Medical Records
}

\author{
Elana Horwitz, $B A^{1 *}$, Bryan Lao, $M D^{2}$, Gregg Robbins-Welty, $M D, M S^{2,3}$, Andrew Tuck, $M D^{2}$ \\ and Jane Gagliardi, $M D, M H S^{2,3}$
}

${ }^{1}$ School of Medicine, Duke University School of Medicine, Durham, North Carolina, USA

${ }^{2}$ Department of Psychiatry and Behavioral Sciences, Duke University Medical Center, Durham, North Carolina, USA

${ }^{3}$ Department of Medicine, Duke University Medical Center, Durham, North Carolina, USA

\begin{abstract}
A 31-year-old woman with a nursing background and loin pain-hematuria syndrome undergoes a renal auto- transplant. During her recovery and prolonged hospitalization, her care team becomes increasingly concerned about a factitious disorder. With the roll-out of the $21^{\text {st }}$ Century Cures Act Final Rule, which involves direct sharing of medical records with patients in real time, multiple inpatient care teams leave their clinical suspicion out of their progress notes for fear of damaging the therapeutic relationship. This case provides an opportunity to consider implications of the Final Rule policy of "open notes" for patients with sensitive psychiatric diagnoses, highlighting the need for effective communication and collaboration between inpatient teams and realistic approaches to the integration of documentation in patient care.
\end{abstract}

\section{Introduction}

In 2016, expansive healthcare legislation known as the $21^{\text {st }}$ Century Cures Act was passed in the United States. This law included the Final Rule or what is commonly called the "Open Notes Rule," which went into effect on April 5, 2021. The legislation shifts ownership of medical documentation from the health system to the patient, with resultant transition of patient access to clinical notes and other health records from a request-based system to immediate electronic access. While Open Notes have already been common practice in at least 10 other countries and the Veterans Affairs network for the last decade, the "Open Notes Rule" expands access to clinical notes, including psychotherapy notes, for all patients. While many clinicians recognize that the ruling may improve patient participation, self-advocacy, healthcare literacy, medication adherence, and even accuracy in charting; at the same time, unintended negative impacts on patient outcomes are of concern [1]. Hypothesized patient groups at high risk of harm from Open Notes include adolescent patients, patients with history of domestic violence, and psychiatric patients [2]. For these reasons, the Open Notes rule allows clinicians to block a note from appearing in the patient-facing records through eight exceptions, which include protecting patient privacy, protecting information security, and preventing harm to a patient or another person [3].

In this case, we present a patient with chronic pain secondary to loin pain-hematuria syndrome (LPHS) along with suspected comorbid factitious disorder who openly viewed and discussed her electronic medical record clinical notes during her hospitalization. Factitious disorder is characterized by the falsification of symptoms for internal reward. Loin pain-hematuria syndrome is a rare condition characterized by chronic bilateral or unilateral flank pain and recurrent hematuria, hypothesized to have a psychogenic component [4]. While literature exists on the effects of the Open Notes Rule in the outpatient setting, little attention has been given to the changing dynamic in the inpatient care setting or to the effect on patient care when sensitive psychiatric information is relevant [5-7]. We will highlight the effects of Open Notes on team communication, treatment planning, and clinical outcome in this patient with factitious disorder, as well as potential strategies to address these unique dynamics.

\section{Case Presentation}

A 31-year-old emergency department nurse, three months postpartum with her first child, with a history of

*Corresponding author: Elana Horwitz, BA, School of Medicine, Duke University School of Medicine, Durham, North Carolina, 100 Lake Ridge PI, Chapel Hill, NC 27516, USA, Tel: 919-6192591, ORCID: 0000-0003-3370-0954

Accepted: November 08, 2021

Published online: November 10, 2021

Citation: Horwitz E, Lao B, Robbins-Welty G, et al. (2021) Open Notes and Closed-Loop Communication: A Case of Factitious Disorder in the Age of Transparent Medical Records. J Psychiatry Treat Res 3(1):74-76 
Citation: Horwitz E, Lao B, Robbins-Welty G, et al. (2021) Open Notes and Closed-Loop Communication: A Case of Factitious Disorder in the Age of Transparent Medical Records. J Psychiatry Treat Res 3(1):74-76

attention deficit hyperactivity disorder (ADHD) and postural orthostatic tachycardia syndrome (POTS) presented to the emergency department with acute on chronic right flank pain, nausea, and vomiting. Medications on presentation included mixed amphetamine salts, hydromorphone, and oxycodone. Review of records over the two preceding years revealed 21 presentations to numerous hospitals with acute flank pain and hematuria. In light of her refractory symptoms and extensive yet unremarkable work-up, numerous teams were already considering the diagnosis of LPHS. She was admitted to the general medicine service for management of refractory pain and inadequate oral intake.

Work-up during admission, including gallbladder and renal ultrasounds, was unremarkable. Pain was alleviated briefly with a percutaneous renal hilar block. The patient subsequently declined renal denervation while reporting worsening pain, for which she received escalating doses of patient-controlled analgesia (PCA) with hydromorphone, thoracic epidural bolus injections, and oral oxycodone. Ultimately, definitive treatment of her LPHS was attempted by renal auto-transplant on the fourteenth day of admission.

Over nearly two ensuing weeks the primary team observed behaviors concerning for factitious symptoms, frequently temporally associated with expected discharge dates, including amassing hot packs (in the same timeframe as multiple unexplained fevers and consistently negative infectious work-ups); hoarding pain medications; tampering with her IV; holding her breath during blood oxygenation readings; and a new-onset unexplained syncopal episode. While a few concerns regarding factitious or malingered symptoms were documented in hidden notes citing "preventing harm to the patient," the majority were omitted from the patient chart in the setting of uncertainty about the merit of single observations and what would happen if the patient read notes documenting these concerns. In fact, the patient frequently brought up specific excerpts from her notes, describing feeling invalidated about her pain level and making arguments to avoid reductions in the opioid dosing. As a result, her primary team voiced hesitancy to document suspicious behaviors given possible disruption of the therapeutic alliance. Ultimately, with her family's encouragement and firm boundaries, the patient was discharged 54 days after her initial presentation.

The patient re-presented to the emergency department two days after hospital discharge with uncontrolled flank pain and was subsequently diagnosed with Enterococcus faecalis bacteremia. Once psychiatry was consulted, the family shared long-held suspicions that she was serially committing intentional self-harm with a goal of remaining hospitalized. They added that previous infectious disease providers had also expressed concerns about possible auto- injection of feces. Following completion of antibiotic treatment, a family meeting was held with the patient, during which time observations and evidence from the family members and hospitalization were shared. The patient revealed feeling unwanted and neglected at home while her husband was busy caring for their newborn but continued to deny intentional self-harm and refuse psychotropic medications.
She was discharged with referrals for outpatient mental health follow-up.

\section{Discussion}

While clinicians may express concern about disruptions to the therapeutic alliance, many patients articulate significant increases in satisfaction associated with the "Open Note Rule" $[7,8]$. In fact, Open Notes can be associated with improved medication adherence in patients with serious mental illness and even improved trust between patients and providers [9]. The Open Notes Rule may help clinicians recognize that medical documentation belongs to the patient, as opposed to the clinician, which could bridge communication gaps, and allow for the development of new, clear and effective communication strategies between clinicians and their patients.

In the case we describe, some clinicians strategized by employing the exclusion criteria "preventing harm to the patient", operating on the premise that if the patient were to read their honest considerations, she might engage in additional self-harming behaviors and thereby come to physical harm. This allowed for the clinicians to hide their mounting concerns in a separate note, while also ensuring clear documentation for gathering evidence towards a differential diagnosis. The majority, however, left observations out of documentation entirely. Given many clinicians have come to rely on the electronic health record as the main source of communication between providers, omitting information from documentation may add to diagnostic uncertainty in situations of suspected factitious disorder. We observed this in this case in which escalating pain medication and additional procedures were prescribed. On the other hand, violations of the $21^{\text {st }}$ Century Cures Act in utilizing exclusions too liberally, when viewed through the lens of the patient as owner of his or her own medical documentation, may even constitute a form of modern day injustice, barring the patient from information to understand their illness [10].

It may be helpful to consider ways in which the Open Notes Rule can serve as a tool for therapeutic intervention, specifically with respect to factitious disorder [11]. Specific note-writing behaviors, such as highlighting patient strengths and reinforcing progress toward shared goals can improve patient trust in their clinicians, and clinicians can benefit from trainings to optimize their use of medical records with respect to patient- centered communication $[12,13]$. Considering that a successful approach for ultimately "confronting" patients with factitious disorder is one that allows for the patient to "save face," indirect communication via open notes may prove to be advantageous, allowing the clinician to express their doubts and the patient a way out of their deception. Developing a strategy to approach note-writing in factitious disorder may provide clinicians with a useful clinical tool to position themselves as patient allies and improve adherence to treatment plans for better outcomes [14]. Overall, optimizing effective communication between members of a multidisciplinary team may involve face-to-face communication for review of complicated medical and psychiatric diagnoses. In light of new rules and interpretations, additional research 
is needed to elucidate the effects and optimize the benefits of the Open Notes Rule on care delivery and outcomes in the inpatient setting.

\section{Disclosure of Funding}

No financial disclosures.

\section{References}

1. DesRoches CM, Bell SK, Dong Z, et al. (2019) Patients managing medications and reading their visit notes: $A$ survey of opennotes participants. Ann Intern Med 171: 69-71.

2. Schapiro NA, Mihaly LK (2021) The 21st century cures act and challenges to adolescent confidentiality. J Pediatr Health Care 35: 439-442.

3. Cures act final rule information blocking exceptions. HealthIT. gov.

4. Smith HS, Bajwa ZH (2012) Loin pain hematuria syndromevisceral or neuropathic pain syndrome? Clin J Pain 28: 646-651.

5. Denneson LM, Chen JI, Pisciotta M, et al. (2018) Patients' positive and negative responses to reading mental health clinical notes online. Psychiatr Serv 69: 593-596.

6. Denneson LM, Cromer R, Williams HB, et al. (2017) A qualitative analysis of how online access to mental health notes is changing clinician perceptions of power and the therapeutic relationship. J Med Internet Res 19: e208.
7. DesRoches CM. Leveille S, Bell SK, et al. (2020) The views and experiences of clinicians sharing medical record notes with patients. JAMA Netw Open 3: e201753.

8. Pamela Peck JT, Meghan Shanahan, Alan Fossa, et al. (2017) Patient access to electronic psychiatric records: A pilot study. Health Policy and Technology 6: 309-315.

9. Blease CR, Walker J, Torous J, et al. (2020) Sharing clinical notes in psychotherapy: A new tool to strengthen patient autonomy. Front Psychiatry 11: 527872.

10. Blease C, Salmi L, Rexhepi H, et al. (2021) Patients, clinicians and open notes: information blocking as a case of epistemic injustice. J Med Ethics.

11. Smith CM, Stavig A, McCann P, et al. (2021) "Let's talk about your note": Using open notes as an acceptance and commitment therapy based intervention in mental health care. Front Psychiatry 12: 704415.

12. Cromer R, Denneson LM, Pisciotta M, et al. (2017) Trust in mental health clinicians among patients who access clinical notes online. Psychiatr Serv 68: 520-523.

13. Dobscha SK, Kenyon EA, Pisciotta MK, et al. (2019) Impacts of a web-based course on mental health clinicians' attitudes and communication behaviors related to use of opennotes. Psychiatr Serv 70: 474-479.

14. Sylvia LG, Hay A, Ostacher MJ, et al. (2013) Association between therapeutic alliance, care satisfaction, and pharmacological adherence in bipolar disorder. J Clin Psychopharmacol 33: 343-350. 